\title{
BRAF Mutation Analysis Was Performed
}

National Cancer Institute

\section{Source}

National Cancer Institute. BRAFMutation Analysis Was Performed. NCI Thesaurus. Code C160333.

An indication that BRAF mutation analysis was performed during the study. 